\title{
First Experience in the Mass Production of Components for the LHC Dipoles
}

\author{
P. Fessia, C. Lanza, D. Perini, and T. Verbeeck
}

\begin{abstract}
This paper reports on the manufacturing features and difficulties experienced for the preliminary mass production of the main mechanical components of the dipole cold mass. The production of about $600 \mathrm{~km}$ of superconducting coil copper wedges, 5000 coil layer jump spacers and boxes, 12500000 austenitic steel collars and 5800000 low-carbon yoke laminations is spread over 4 European countries and involves 6 manufactory firms.

The general technical requirements for the manufacturing process as well as the imposed production checks and quality controls are reviewed. An overview of the preliminary results is presented with an outlook toward the analysis and statistical which are in a process to be implemented for the follow-up of the mass production.
\end{abstract}

Index Terms-S.C. Dipole components.

\section{INTRODUCTION}

$\mathbf{T}$ HE MASS production of the main mechanical components for the 1248 LHC main dipoles involves 4 different European countries and consist in about $600 \mathrm{~km}$ of superconducting coil copper wedges, 5000 coil layer jump spacers and boxes, 12500000 austenitic steel collars and 6000000 lowcarbon yoke laminations.

The production technologies of these components are very different spanning from cold-drawn copper profile to precise machining of glass fiber charged epoxy resin to fine-blanking of metal coils or sheets. Nevertheless the quality control and production follow-up is very similar and most of our efforts concentrated to find standard manufacturing process controls and procedures.

The geometry of these components is critical for the correct assembly of the cold masses and contributes to attain the required stringent tolerances in field quality. In particular, for the precise positioning of the cables in the superconducting coils the copper wedges are critical. The correct position of the coil layers and blocks will be maintained by the collars that surround and compress the coils. The yoke laminations will contribute to the accuracy of the mechanical assembly and to the fine-tuning of the magnetic length.

\section{QUALITY CONTROL}

To follow a mass production it is necessary to analyze a huge amount of data concerning the process. All these data are contained in the so-called "Traceability files." ISO 9000 defines

Manuscript received September 24, 2001.

The authors are with the Main Magnet and superconductor Group, LHC division, CERN, 1221 Geneva 23, Switzerland (e-mail: \{Paolo.Fessia; Cristiano.Lanza; Diego.Perini; Theodore.Verbeeck\}@ cern.ch).

Publisher Item Identifier S 1051-8223(02)04173-8. traceability as: the ability to trace the history, application or location of an entity by means of recorded identifications.

Two useful tools to organize and manage the necessary data are the workflow and the ABS (Assembly Breakdown Structure) [1], [6]. The workflow shows all the phases of a production process and in which position, during the process, the data are collected in a "traveler" (Fig. 1). The ABS shows the history of an entity passing trough different processes.

The results of the measurements are usually recorded on standard EXCEL ${ }^{\circledR}$ spreadsheets before being automatically loaded in a centralized ORACLE ${ }^{\circledR}$ database structure designed with a Web interface application.

The follow-up of the production must be based on a powerful statistical analysis program. Not often this kind of analysis is available in all the manufacturing firms and it imposes therefore the data to be analyzed off-line resulting in delays in the corrective reaction time over the production process. The used approach rely mostly on graphical analysis of the data:

- a graphical representation of the measurements as a function of time to monitor the trend and eventually to detect drifts or shifts of the recorded data;

- a histogram with the frequencies of the measurements to verify the distribution of the data.

\section{COPPER WEDGES}

\section{General}

The series production of the copper wedges consists of a supply of about $600 \mathrm{~km}$ of superconducting coil copper wedges with four different profiles. One dipole cold mass contains approximately 114 meters of copper wedges for each profile.

As mentioned in the introduction, the quality of the magnetic field is given by the accurate position of the cables in the superconducting coils. The correct position of the cables is achieved by inserting the insulated copper wedges during the winding of the coils to separate different blocks of cable.

To obtain the desired field quality, very tight geometrical tolerances are imposed: a few hundredths of millimeter for critical dimensions. The cold-drawn copper wedges are shaped at room temperature from round copper profile beams which are forced through a precise die. The wedges are delivered in $3.6 \mathrm{~m}$ long pieces.

\section{Acceptance Criteria for the Production Processes}

The cross-section dimensions of the copper wedges are measured with a spherical probe using a three-dimensional computer-controlled scanner. The dimensions have to be checked 


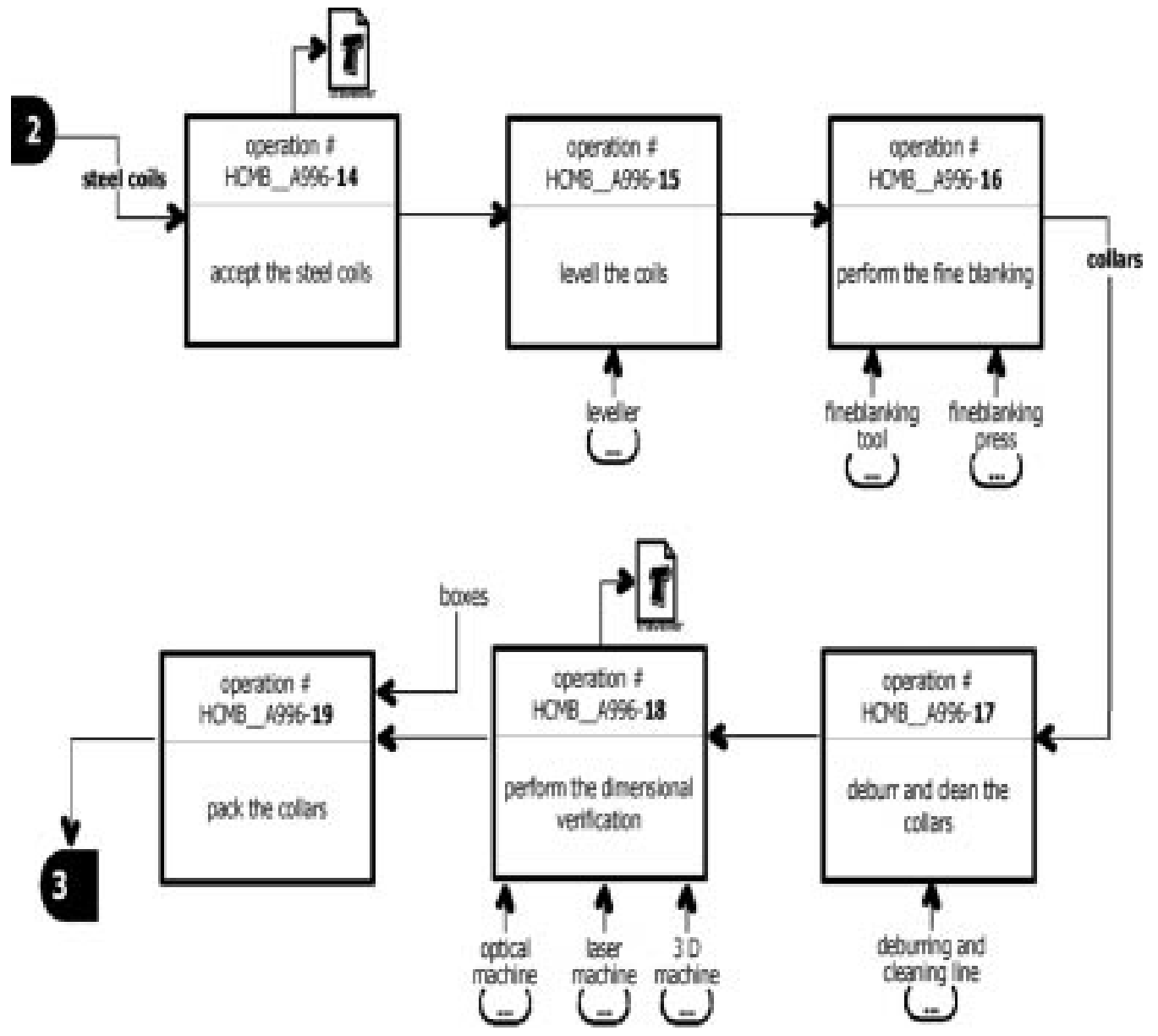

Fig. 1. Example of a workflow for the collar production.

in at least three sections evenly spaced along the same copper wedge.

At the beginning of the series production, the geometrical checks were carried out three times per batch (for a profile, a batch is the quantity necessary to assemble ten dipoles): the first, the middle and the last copper wedge of the batch. From batch number 11 onward, for each profile, only the middle copper wedge produced in a batch will be checked.

\section{Analysis}

In order to monitor the production and according to the quality control requirements presented in the introduction, the collected data for the copper wedges are represented graphically as shown in Fig. 2.

In this case, a shift is clearly visible in the mean of the production; although all the values remain well inside the interval of specification, the origin of the behavior and the implications on the coil assembly performance may easily be investigated. The small shift which can be seen is mainly due to a new manufacturing lot after a production stop of about 2 months.

\section{LAYER JUMP SPACERS AND BOXES}

\section{General}

The electrical connection between the inner layer coil and the outer layer coil in the LHC dipoles is called layer jump

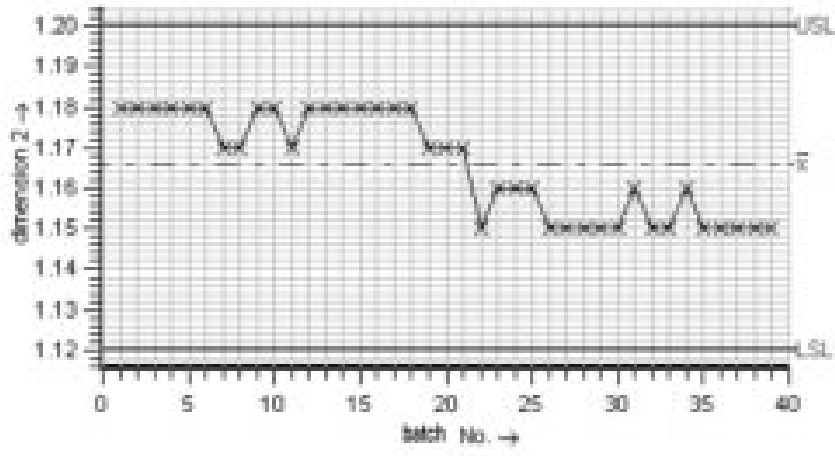

Fig. 2. Measurement trend of a batch series of the type 1 copper wedge (USL: upper specification limit; LSL: lower specification limit).

and splice. The layer jump and splice is a complex 3-D region housed in special collars. Tapered plastic elements, called layer-jump spacers, are placed in contact with the coils. The " $\mathrm{S}$ " shaped inner layer cable, going in radial direction to the outer layer, is kept in position by the so called layer-jump box. For the complete LHC dipole production, we need 10000 layer-jump spacers of two different profiles and 5000 layer-jump boxes. As these elements are in contact with the coils, it is essential that their tolerances be comparable to those given for the coil geometry: $\pm 20 \mu \mathrm{m}$. 

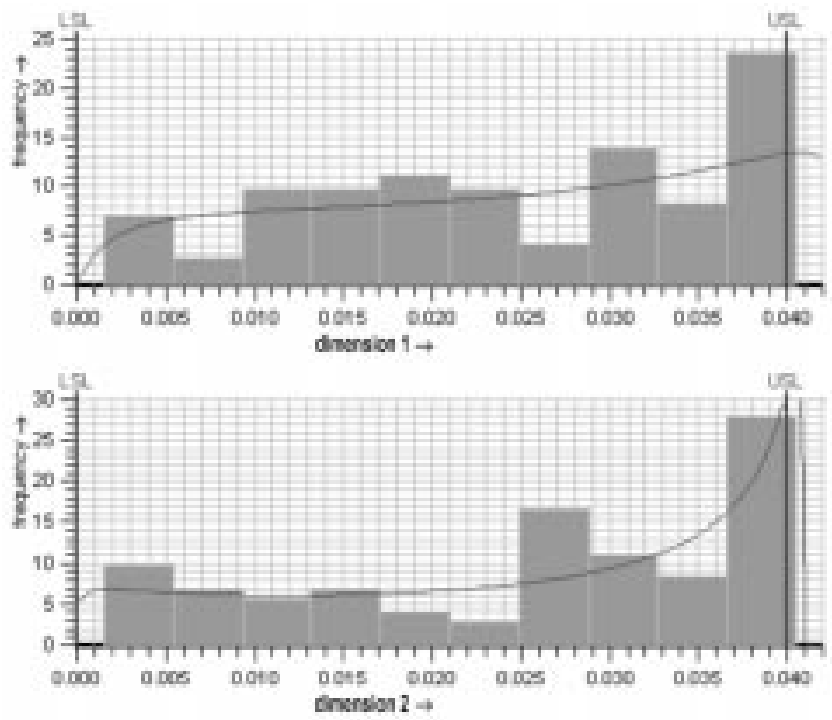

Fig. 3. Results of measurements on two different dimensions for layer jump spacer production.

The layer-jump spacers are machined from glass-fiber charged epoxy tubes. The support tooling during cutting has been carefully studied. Experimentally, it was shown that the force exerted by the cutter during the machining was sufficient to bend the piece if not properly kept and hence to produce pieces outside the tolerances.

The layer jump box is molded in ULTEM ${ }^{\circledR} 2300$ (30\% glassfiber charged $\mathrm{ULTEM}^{\circledR}$ ). Then the "S"-shaped groove is machined.

\section{Acceptance Criteria for the Production Processes}

All the parts are checked for their geometry. The dimensions have to be validated for at least three sections along each piece.

For the measurements, special jigs were manufactured. The piece is fixed onto the jig and checked with transducer touch gauges in pre-defined locations. The zero is made using calibrated steel pieces. The use of dedicated jigs allows the precise measurement of each piece and only a few seconds are necessary to measure one section.

\section{Analysis}

Although the delivered layer jump spacers are all within tolerances, the analysis outline shows two Gaussian distributions overlapped with the most frequent values close to the upper and lower acceptance levels (Fig. 3). This may be explained by the high rejection rate of the produced parts and, in particular, to the tight tolerances to be achieved which are nearly at the limit of the state of the art for machined charged plastics.

In order to increase the acceptance and the production rates, the manufacturer has now started to implement some changes in the tooling design and to optimize the production.

\section{Collars AND Yoke LAMinations}

\section{General}

The production of high-strength austenitic-steel collars [2] and low-carbon steel half-yoke laminations and inserts [3] involves the supply of respectively 12500000 and 6000000 pieces. A dual sourcing policy for both supplies was applied so as to cope with eventual delivery or manufacturing difficulties. In total three fine-blanking manufacturers located in Europe are involved in these supplies.

High precision and tight tolerances are required for critical dimensions (typically $\pm 0.02 \mathrm{~mm}$ ) and are obtained with a precision fine-blanking process.

\section{Tools}

For the collars, there are a total of six different configurations to be manufactured. For the standard yoke laminations, one type only is required while four different types are necessary for the cold mass yoke extremities. Considering the large number of elements to be made and the tight tolerances involved, a modular structure of the blanking tool allowing easy re-sharpening and corrective actions on critical parts was requested. One contractor favored a single tool for the two collar types while the other made two standard separated tools. After the fine-blanking process, the pieces must be carefully deburred, cleaned and, for the low-carbon yoke laminations, protected against oxidation. An average of three to four tool corrections were necessary before tolerances could be attained. Many more corrections were needed for both collar types made with a single tool.

\section{Acceptance Criteria for the Production Processes}

The tool acceptance was granted after a pre-series of $100 \mathrm{col}-$ lars and yoke laminations had shown that, for two successive samples every ten produced, the geometrical dimensions measured with a 3-D computer-controlled scanner were within tolerances (type A checks). During the series production, two dimensional check levels were imposed:

- the low-frequency level, using a 3-D scanner on one piece every 2500 pieces produced

- the on-production level, check every 500 pieces produced, using a laser beam scanning machine (type B checks).

The rates of these checks will be decreased by a factor three after the first 90 dipoles have been assembled.

The imposed check points are related to the most critical regions involved for the good functionality of the assembled parts. As an example, Fig. 4 is showing the location of the imposed check points for the yoke lamination production.

Another checking method, based on a smart-fit technique, is mainly used during the initial phase of the tool optimization or to get a good image of the complete profile of a produced piece.

Based on a precise CCD camera, the method is inspecting the piece to be measured. A software program is performing a best fit with respect to fixed reference points and comparing the results with the drawing file. Fig. 5 shows a result for a collar piece, the boundaries for the tolerances are visualized and the regions outside the tolerances are identified and evaluated. 


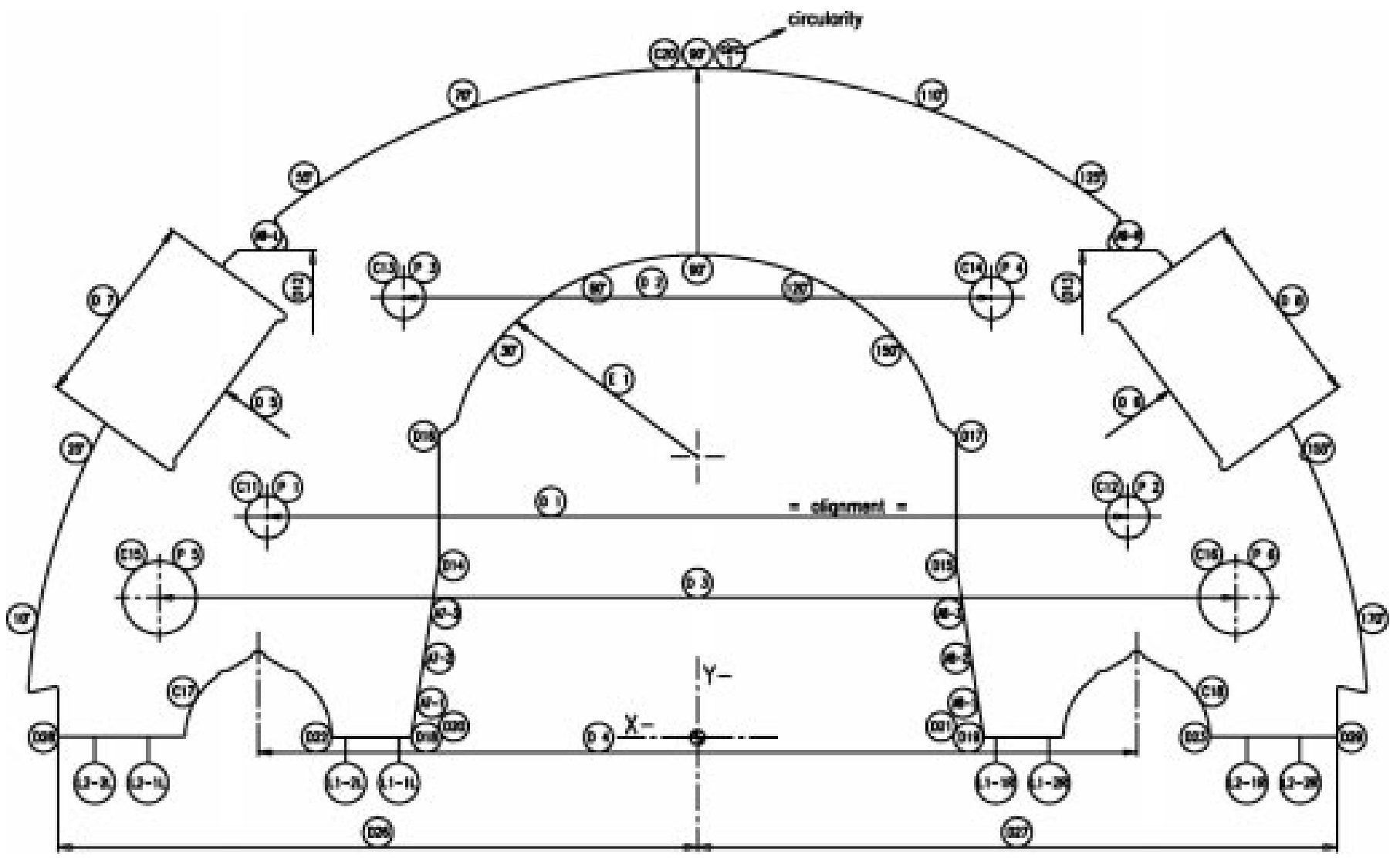

Fig. 4. Low-carbon yoke lamination with location of check points.

\section{Acceptance Criteria for the Production Processes}

The criteria assessment was defined after statistical deviations measurements (e.g., Gauge R\&R study [5]) had been made repeatedly on a same collar and on consecutive collars. The criteria was set as follows: the batch (one batch is equivalent to the total number of fine-blanked pieces necessary for one dipole) is accepted if the mean value recorded on the three level A checks are within the tolerances. When the inspection frequency will be released to only one check per batch (after the production of 90 cold masses), a floating mean of the current batch and the two previous ones will be considered.

\section{Implemented Analysis Means}

From a Web application written in Java script, a data selection facility combined with an interactive drawing allows a visualization of the measuring points to be analyzed. Based on this concept, a powerful statistical facility including standard parameters is available (Fig. 6). Covering the selected samples, a summary table for the maximum, minimum, average, and standard deviation of the selected points are shown; a moving average facility can also be displayed [4].

For each batch produced, the drift of the recorded checking points are closely followed-up and, as soon as required, preventive tool re-sharpening operations are programmed to recover the initial boundaries of the tolerances. Based on the present experience, a tool re-sharpening is made after about 30000 press strokes for both the collar and yoke lamination productions.

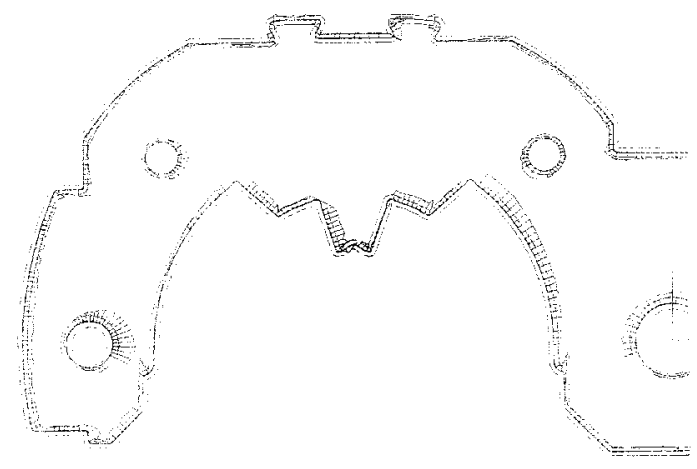

Fig. 5. Smart-fit plot for a particular of a collar. The outs of tolerance were corrected during the tool optimization.

\section{CONCLUSIONS}

The series production of different components used in the superconducting LHC dipoles has started. We have used the first year to settle and to clarify the production and quality control techniques. As now the major problems for producing large scale pieces are practically solved, there remain some improvements to be implemented to increase the production rate and to reduce the larger spread in dimensions observed for certain pieces.

Finally, it must be stressed that the mass production of fourteen different parts produced in four different countries by six different firms and using in most cases different techniques have been all monitored and analyzed with the same standard approach. 

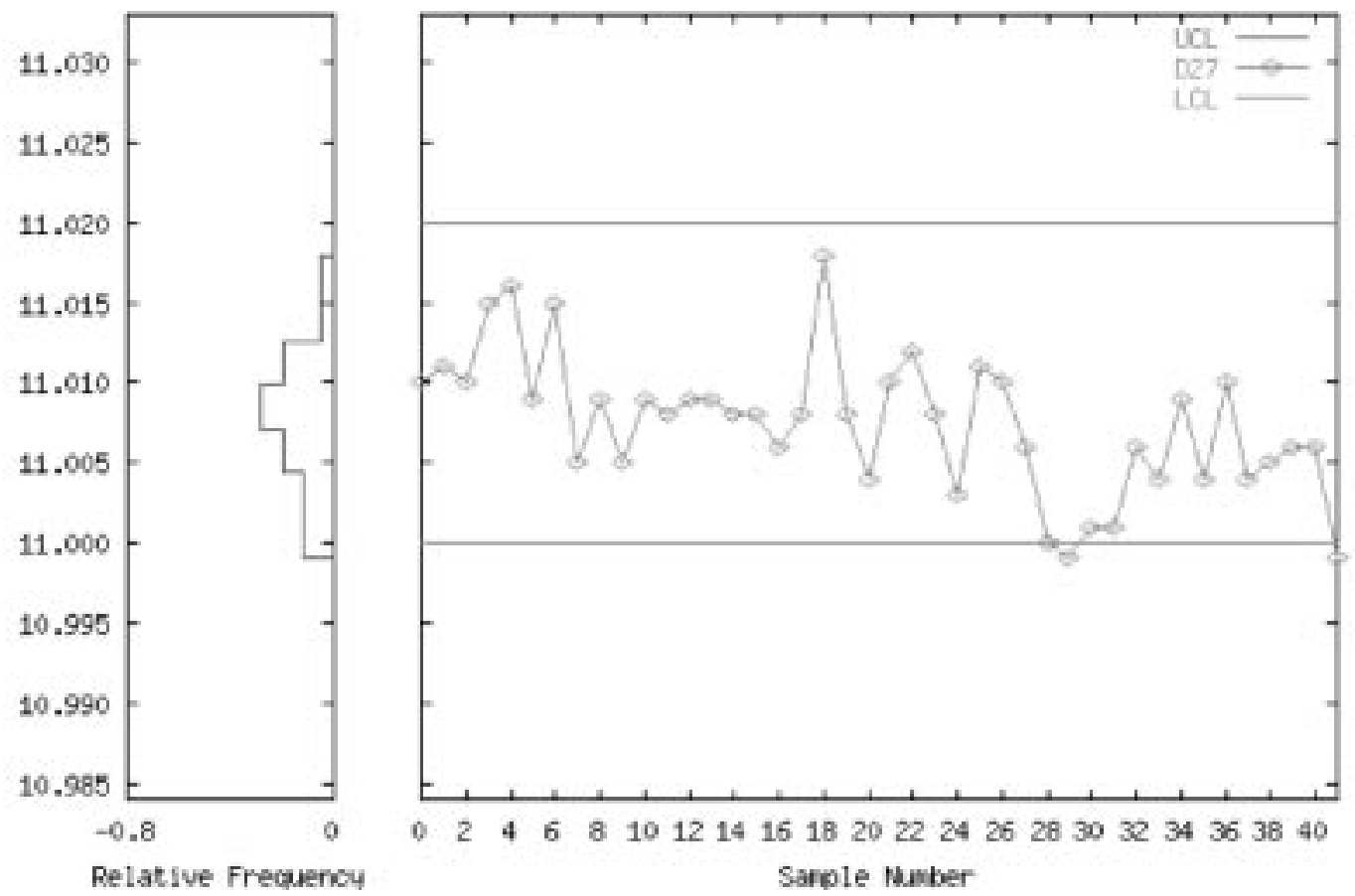

Fig. 6. Example of a statistical web application (see text for detail).

\section{ACKNOWLEDGMENT}

The authors would like to thank the staff of the six firms who are supplying the parts described in this note. In particular, we wish to thank G. Malvestiti (Malvestiti, Italy), G. Biana (Elay, Spain), G. Vezzaro (O.M.A, Italy), F. Tosti and G. Franceschelli (Tosti, Italy), V. Iljanko, E. Oikarainen and I. Vuoristo (Outokumpu, Finland); without their valuable expertise and collaboration this work would not have been possible. Finally, they would like to thank R. Perin, J. Vlogaert, and C. Wyss for their continuing encouragement throughout this large scale production project.

\section{REFERENCES}

[1] "LHC Quality Assurance Plan no. LHC-PM-QA-302.00,", to be published.

[2] C. Lanza and D. Perini, "Characteristics of the austenitic steels used in the LHC main dipoles," IEEE Trans. Appl. Superconductivity, submitted for publication.

[3] S. Babic, S. Comel, F. Beckers, F. Brixhe, G. Peiro, and T. Verbeeck, "Toward the production of 50000 tonnes of low-carbon steel sheet for the LHC superconducting dipole and quadrupole magnets," IEEE Trans. Appl. Superconductivity, submitted for publication.

[4] J. Beauquis and J. Miles, "Data analysis and geometry measurement results for LHC dipole components,", CERN/LHC-MMS Divisional Report, to be published.

[5] "Measurement systems analysis (MSA) reference manual," Chrysler Corporation, Ford Motor Company, General Motors Corporation.

[6] P. Bonnal, "private communication," unpublished. 